\title{
COMPUTATION AND EXPERIMENTAL COMPARISON OF THE DEFORMATION BEHAVIOR OF PANTOGRAPHIC STRUCTURES WITH DIFFERENT MICRO-GEOMETRY UNDER SHEAR AND TORSION
}

\author{
Hua Yang, Wolfgang H. Müller \\ Technische Universität Berlin, Institute of Mechanics, Berlin, Germany \\ e-mail: hua.yang@campus.tu-berlin.de
}

\begin{abstract}
Additive manufacturing methods, commonly known as 3D printing, allow more sophisticated designs to be created. Willingly designed substructures incorporated into the solid open up new possibilities for uncommon macroscopic deformation behavior. Such a man-made structure is also referred to as a metamaterial. A detailed simulation of a polymer-based metamaterial is challenging but accurately possible by means of the theory of elasticity. In this study, we present experimental investigations of a metamaterial composed of pantographic substructures of different internal geometry. The pantographic structures show an unexpected type of deformation, which can be modeled via elasticity with the aid of direct numerical simulation by using the Finite Element (FE) method. In other words, a detailed mesh is generated involving the substructure. The metamaterial is additively manufactured out of a common polymer showing nonlinear elastic deformation and, therefore, hyperelastic material models are used. Specifically, analytical solutions of a circular cylinder are examined and compared in the case of extension and torsion in order to comprehend the effects of the coefficients inherent to the energy function of the hyperelastic model. Finally, FE computations of pantographic structures are performed and compared with the experimental measurements.
\end{abstract}

Keywords: nonlinear elasticity, metamaterial, numerical simulation

\section{Introduction}

Additive Manufacturing (AM) provides an advanced manufacturing technique that allows one to fabricate sophisticated geometry prototypes at a rapid pace and relatively low cost, which makes the fabrication of complex metamaterials possible. Metamaterials are materials which are architectured from so-called "microscopic" elements; by tailoring these micro-elementary constituents or substructures, the macroscopic deformation behavior can be modified completely. Hence the design of a functionalized material for specific purposes leading to novel types of deformation patterns becomes possible (Del Vescovo and Giorgio, 2014; Barchiesi et al., 2018b). As it is commonly known, a material in continuum mechanics is scale-invariant, i.e., it responds to an applied force in the same way on every length scale. However, by constructing a metamaterial with a substructure on the micrometer length scale, we observe different deformation responses than can be expected on the macro-scale if no substructure is present. In order to simulate the deformation phenomenon on the macro-scale, we can introduce new parameters allowing the material model to fully grasp the new pattern. This method is known under different names, for example, micropolar medium, strain gradient theory, generalized mechanics, couple stress material (Mindlin and Eshel, 1968; Toupin, 1964; Lam et al., 2003; Altenbach and Eremeyev, 2012), for historical remarks see dell'Isola et al. (2014), and for a recent review see Thai et al. (2017). It is important to emphasize that the theory of elasticity is valid and accurate on the micro-scale. The introduction of new structure-related parameters are necessary as a part of a 
homogenization procedure resulting in including higher-order terms in the formulation. As it was mentioned earlier, it is not clear yet how these structure-related parameters can be determined.

The pantographic structure shown in Fig. 1, composed of two orthogonal arrays of beams connected by internal cylinders called pivots, is a metamaterial, which has been investigated by the aforementioned generalized method (dell'Isola et al., 2016). Interestingly, pantographic structures can be deformed without damage up to large deformation and still remain in the elastic reversible range (dell'Isola et al., 2015, 2016). Recently, simple shear and torsion tests (Misra et al., 2018) applied to 3D printed pantographic structures with different combinations of internal geometric parameters of substructures have been conducted and showed highly nonlinear responses as well as some uncommon macroscopic deformations (Barchiesi et al., 2018a; Ganzosch et al., 2016). The macroscopic behavior of the metamaterial is affected by underlying deformation mechanisms related to geometry and various sizes contained within the substructures. For a rigorous understanding of the mechanisms and effects of the substructural properties on the overall behavior of these material systems, we aim at a direct numerical simulation by using the Finite Element Method (FEM), for which an appropriate constitutive law is necessary. The classical Hooke's law assumes a linear stress-strain relationship, which is not accurate enough for the pantographic structures fabricated by polyamide materials, because they are capable of undergoing nonlinear elastic deformation. Therefore, a nonlinear elastic (hyperelastic) constitutive law is needed.

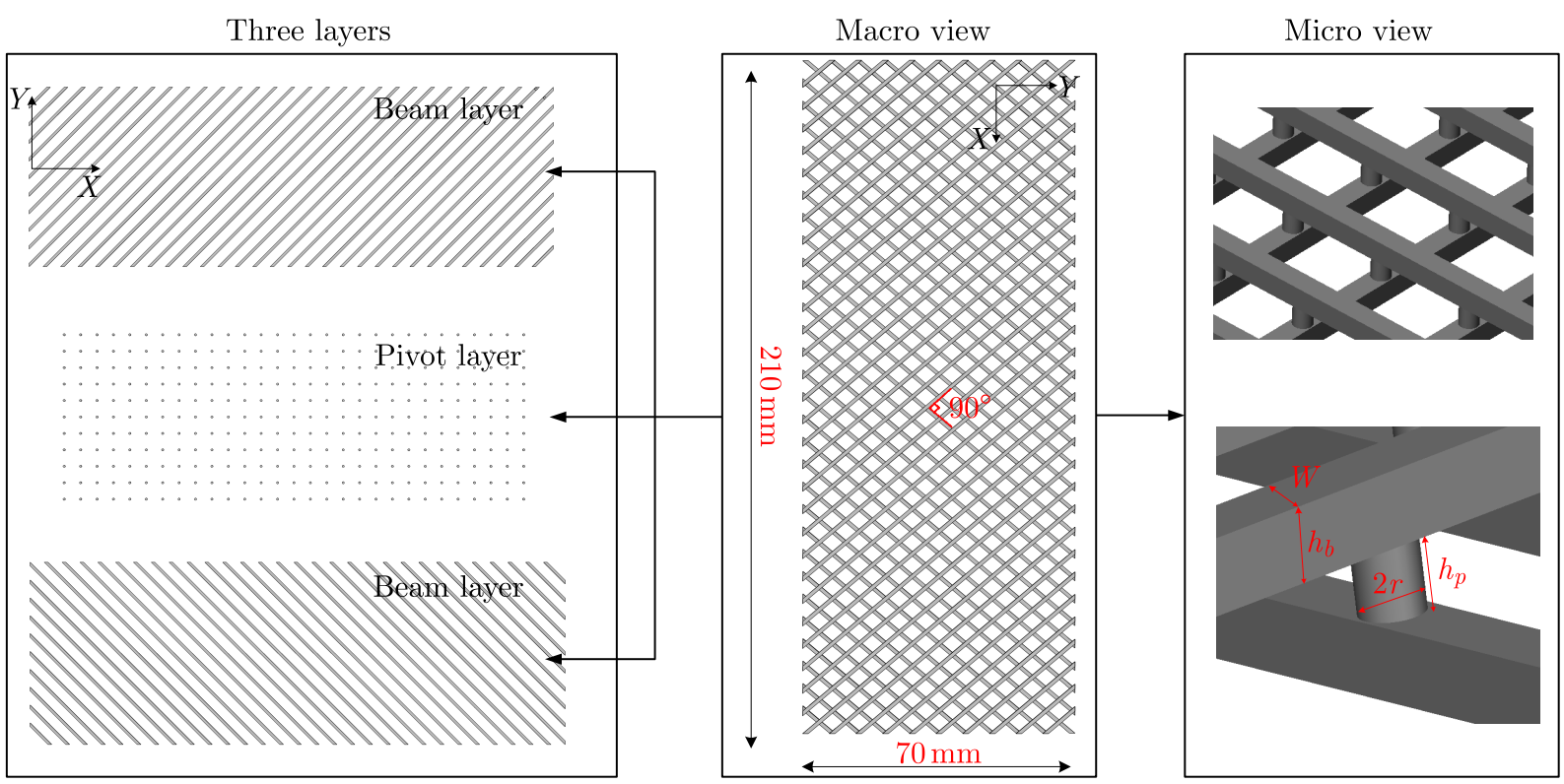

Fig. 1. Topology and geometry of pantographic structures

Over the years, a number of hyperelastic models have been proposed that use specific strain energy density functions for capturing the behavior of various rubber-like materials (Holzapfel, 2000), so that mathematical formulations differ from one to another. But ultimately, it is not only the mathematical form that is important, but also its numerical values representing the behavior of the material. Numerical calculations for rubber-like materials are often troublesome due to the lack of a suitable model capturing the material behavior at large deformations. In the work of Yang et al. (2018), a series of hyperelastic material models were studied and calibrated. The computations also show that the nonlinear material model is in good agreement with experimental data. In this work, we make use of hyperelastic models whose built-in coefficients were calibrated in (Yang et al., 2018) and investigate and compare their performance with regard to different combinations of internal geometry sizes of substructures under shear and torsion experiments of pantographic structures. 
The metamaterial under a mono-axial shear test as well as a torsion test will be described in Section 2. The hyperelastic model will also be described, and the analytical solution of a cylinder under extension and torsion will be investigated. FEM computations are obtained by implementing hyperelastic models with the aid of open-source codes developed under the FEniCS project, see (Logg et al., 2011). A comparison and discussion of the experiments and computations are presented in Section 5.

\section{Experimental setup of the shear and torsion tests}

\subsection{Loading devices}

The simple shear experiments shown in Fig. 2 will be revisited here. For more details, the reader is referred to Ganzosch et al. (2016). The experiment was performed in displacement-controlled manner by using an MTS TYTRON-250 system where a load cell capable to record forces in the range of $\pm 250 \mathrm{~N}$ was used to measure the reaction force. A displacement-rate $(15 \mathrm{~mm} / \mathrm{min})$ was defined for shearing the specimen by means of a DC-linear motor where an air-film-bearing realized almost frictionless movement. In order to reduce the influences of gravity the system was arranged horizontally. The lower part of the sample was fixed to a metal slide situated on the left hand side, cf. Fig. 2. The upper part was connected to the right hand side of a metal slide and driven in parallel to the lower side up to a distance of $\Delta \ell_{\max }=70 \mathrm{~mm}$. The maximum prescribed displacement was of the same size as the transverse length of the sample. Hence the specimen was subjected to large deformations. The displacement-controlled motion of the upper part was defined by a constant displacement-rate of about $0.5 \mathrm{~mm}$ in a linear motion sequence. The machine automatically stopped when the maximum displacement $\Delta \ell_{\max }$ was reached.
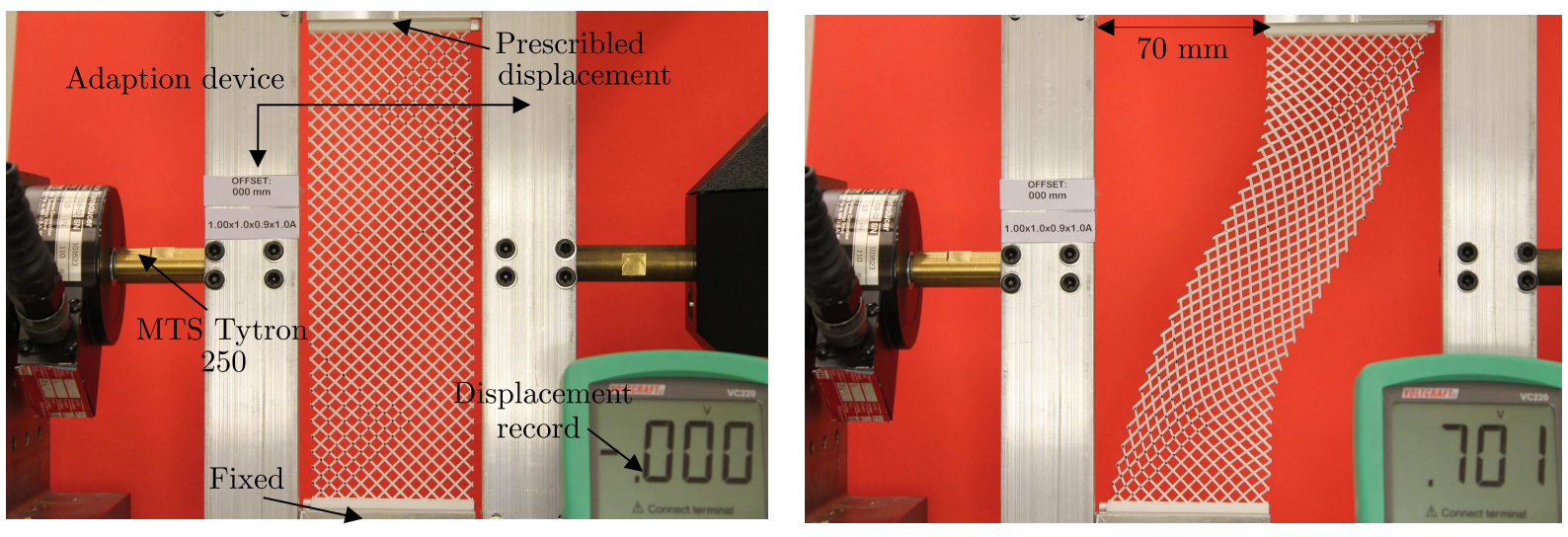

Fig. 2. Pantographic structure subjected to mono-axial shear

The torsion experiment for pantographic structures is illustrated in Fig. 3 and will be briefly discussed in what follows. More details can be found in (Ganzosch et al., 2018). The experiment was conducted using a Zwick-Z010 system equipped with a load cell (Zwick-Serie) capable of measuring and recording the axial reaction force. One side of the sample is fixed, the other side is rotated by $1^{\circ} / \mathrm{min}$ up to $180^{\circ}$. Because of the large resulting rotation angle, the sample is under large deformation. It should be noted that the digital image correlation technique (Brodecki et al., 2018) can be used to investigate the peculiar out of plane deformations. This, however, is out of the scope of this paper.

Three pantographic structures shown in Fig. 4 characterized by four different internal geometrical parameters but equal macroscopic dimensions of $210 \mathrm{~mm} \times 70 \mathrm{~mm}$ were printed layer by layer from polyamide powders by using a laser sintering technique. Table 1 contains dimensions 

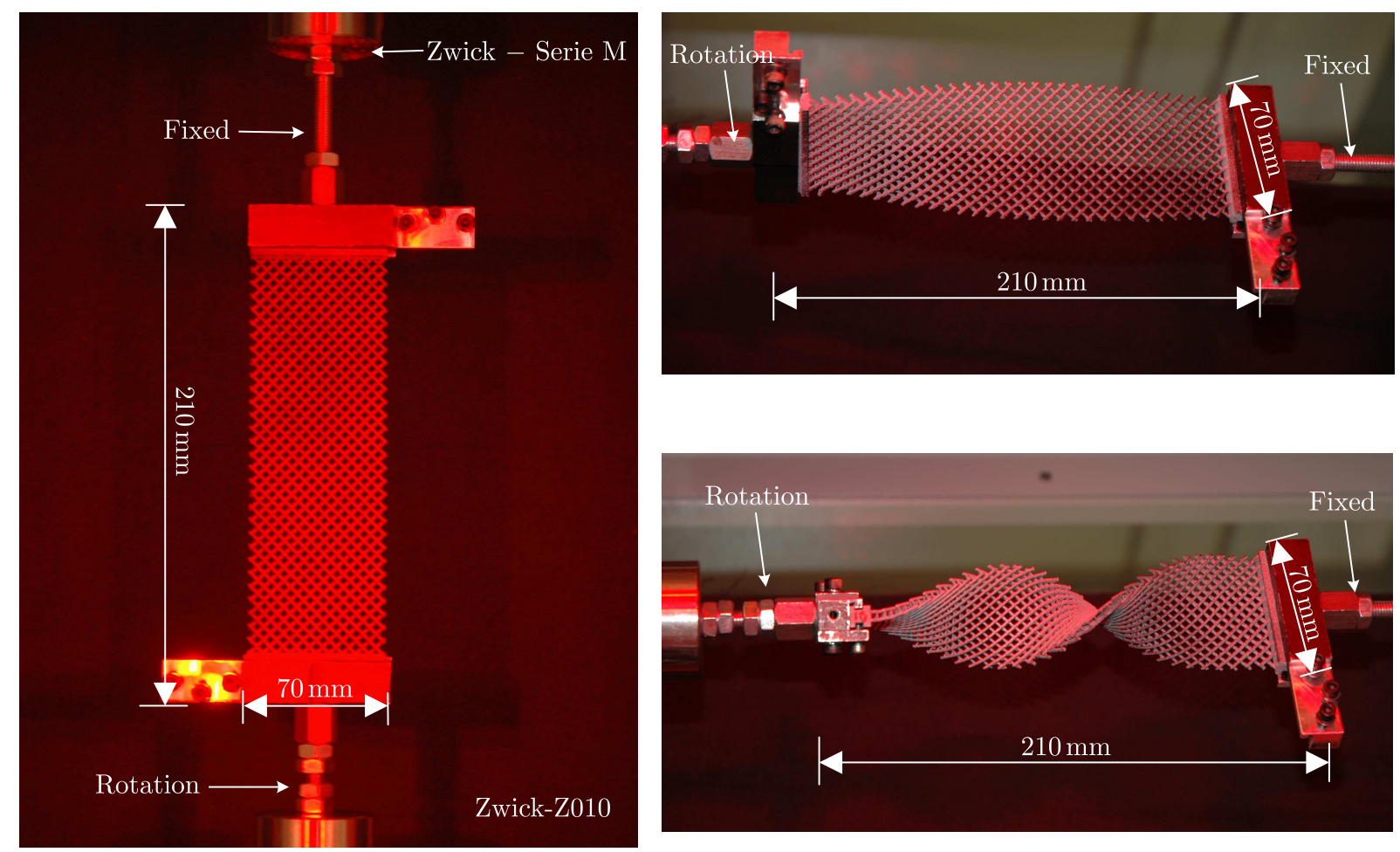

Fig. 3. Pantographic structures under torsion
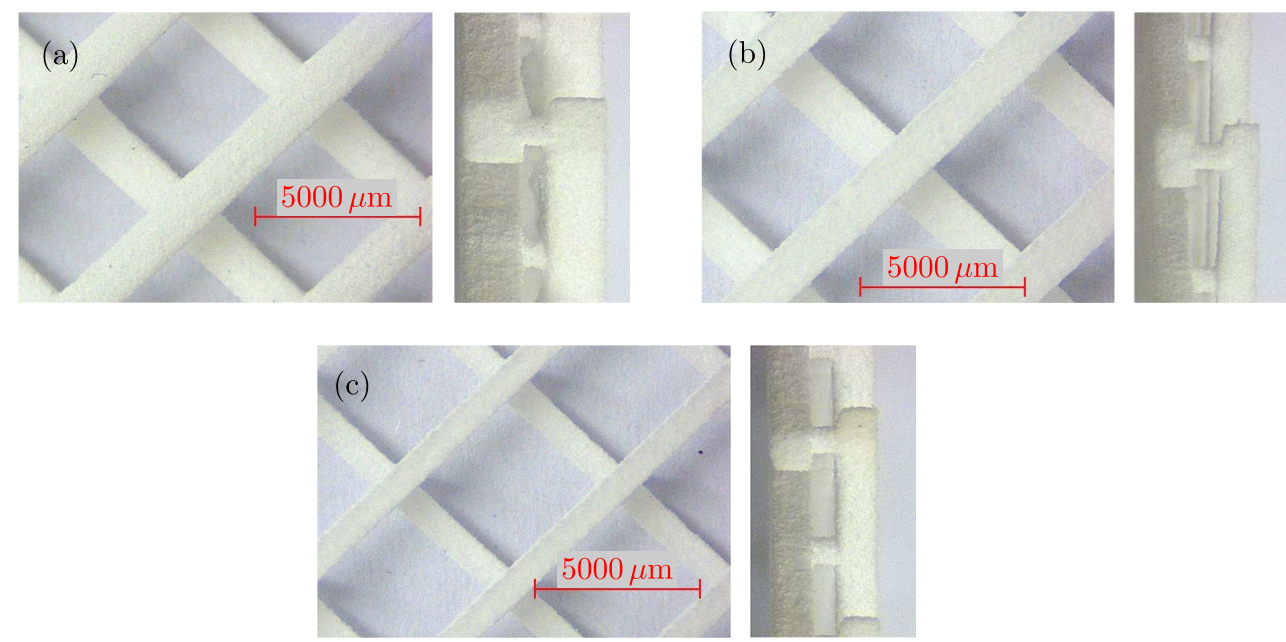

Fig. 4. Samples of pantographic structures made up of polyamide by 3D printing

of the beam width $w$, height $h_{b}$, pivot radius $r$ and height $h_{p}$. The beam dimensions were varied in these specimens with the aim to understand the mechanisms and effects of the substructure properties on the overall behavior of these metamaterials. All of the samples show large nonlinear behavior (more details will be shown in Section 5), exhibit no damage, and the large deformations remain reversible. Therefore, we may say that the elastic behavior dominates in the experiments. The influence of viscoelastic behavior occurring during the experiments seems non-negligible at the first glance, however, its role will be investigated in a future study; for example cyclic loading experiments can be conceived (Kowalewski et al., 2014). Here we expect, by studying and selecting appropriate elastic material models, that one of the constitutive laws is able to capture the peculiar properties of the pantographic structures. 
Table 1. Geometrical characteristics of the microstructure

\begin{tabular}{|c|c|c|c|c|}
\hline Type & $w[\mathrm{~mm}]$ & $h_{b}[\mathrm{~mm}]$ & $r[\mathrm{~mm}]$ & $h_{p}[\mathrm{~mm}]$ \\
\hline \hline A & 1.60 & 1.60 & 0.45 & 1.00 \\
\hline B & 1.60 & 1.00 & 0.45 & 1.00 \\
\hline C & 1.00 & 1.00 & 0.45 & 1.00 \\
\hline
\end{tabular}

\section{Hyperelastic model}

Following the Lagrangian description of kinematics of continua, the position of a material particle is denoted by $\mathbf{X}$ in the reference configuration. Moreover, the position of the material particle is given by $\mathbf{x}$ in the current configuration due to a certain mechanical loading. According to the notation of classical continuum mechanics, the deformation gradient tensor $\mathbf{F}=\partial \mathbf{x} / \partial \mathbf{X}$ is introduced and, subsequently, the right and left Cauchy-Green strain tensors $\mathbf{C}$ and $\mathbf{B}$, respectively, can be obtained $\mathbf{C}=\mathbf{F}^{\mathrm{T}} \mathbf{F}$ and $\mathbf{B}=\mathbf{F F}^{\mathrm{T}}$. Note that $\mathbf{C}$ and $\mathbf{B}$ have the same eigenvalues and principal invariants. The principal invariants represented by $I_{1}, I_{2}, I_{3}$ of the right and left Cauchy-Green strain tensor are defined by (Holzapfel, 2000)

$$
\begin{aligned}
& I_{1}=\operatorname{tr}(\mathbf{C})=\operatorname{tr}(\mathbf{B}) \\
& I_{3}=\operatorname{det}(\mathbf{C})=\operatorname{det}(\mathbf{B})
\end{aligned}
$$

We assume incompressibility leading to

$$
I_{3}=1
$$

In this paper, three invariant based hyperelastic models have been selected, namely the Neo-Hookean model, the Isihara model and the Yeoh model, whose strain energy density functions are listed in Table 2. The Neo-Hookean model is the simplest model and only depends on the

Table 2. Strain energy density function for hyperelastic models

\begin{tabular}{|l|c|}
\hline \multicolumn{1}{|c|}{ Model } & Equation \\
\hline \hline Neo-Hookean & $\frac{1}{2} \mu\left(I_{1}-3\right)$ \\
\hline Isihara & $C_{10}\left(I_{1}-3\right)+C_{01}\left(I_{2}-3\right)+C_{20}\left(I_{1}-3\right)^{2}$ \\
\hline Yeoh & $C_{10}\left(I_{1}-3\right)+C_{20}\left(I_{1}-3\right)^{2}+C_{30}\left(I_{1}-3\right)^{3}$ \\
\hline
\end{tabular}

first invariant $I_{1}$. It is characterized by one parameter only, the shear modulus $\mu$. The Yeoh model, which also depends only on the first invariant $I_{1}$, contains three terms with three material parameters. It has been demonstrated to fit various modes of deformations using the data obtained from uniaxial tests alone (Chen and $\mathrm{Wu}, 1997$; Lawlor et al., 2011). Furthermore, the Isihara model, which is a polynomial function of $I_{1}$ and $I_{2}$, has been selected here. From the mathematical point of view, the Isihara model shows similarities to the Yeoh model. They both comprise the linear and quadratic part of $I_{1}-3$; the major difference is that the Isihara model contains the $I_{2}-3$ part, whereas the Yeoh model does not. The values of inherent material parameters shown in Table 3 are used from Yang et al. (2018). Note that all parameters were calibrated from an axial tensile test. In view of the differences of the mathematical equations of these hyperelastic models, it is even more difficult to interpret the physical meaning of each material parameter in the constitutive laws. These subtle differences may lead to discrepancies in values when predicting the behavior of pantographic structures. In order to understand the possible distinctions, analytical solutions of a cylinder under extension and torsion, shown in Fig. 5, are performed below, for all of the three aforementioned hyperelastic models. Actually, 
Table 3. Coefficients of the hyperelastic models

\begin{tabular}{|l|c|}
\hline \multicolumn{1}{|c|}{ Model } & Coefficients \\
\hline \hline Neo-Hookean & $\frac{1}{2} \mu=83.3 \mathrm{MPa}$ \\
\hline Isihara & $C_{10}=-929 \mathrm{MPa}, C_{01}=1013 \mathrm{MPa}, C_{20}=648 \mathrm{MPa}$ \\
\hline Yeoh & $C_{10}=73 \mathrm{MPa}, C_{20}=-2653 \mathrm{MPa}, C_{30}=64945 \mathrm{MPa}$ \\
\hline
\end{tabular}

such analytical solutions were studied in the poineering work of Rivlin (1948). For the convenience of the reader, the pertinent equations are repeated below for a general strain energy function $W$.
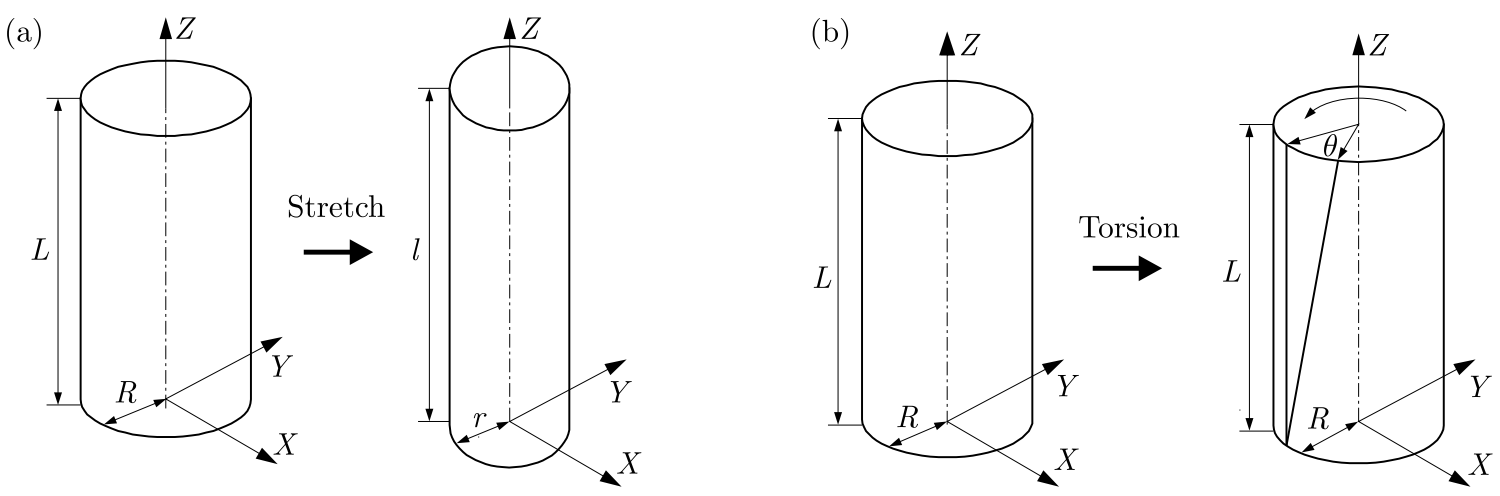

Fig. 5. Schematic of a cylinder under pure stretch (left) and pure torsion (right)

Consider a homogeneous cylinder made of an incompressible hyperelastic material having length $L$ with a circular cross-section with the initial radius $A$. One end of the cylinder is fixed while the other side is subjected to uniaxial displacement and to rotation. Because of the axial symmetry, cylindrical polar coordinates are used with $(R, \Theta, Z)$ in the reference configurations and $(r, \theta, z)$ in the current configurations, respectively. The deformation is expressed by

$$
r=\alpha R \quad \theta=\Theta+\tau \lambda Z \quad z=\lambda Z
$$

where $\alpha$ is the ratio between the deformed and the initial radius; $\lambda$ is the ratio of the deformed and the initial length; $\tau$ is the angle of twist per unit length in the current configuration. The deformation gradient $\mathbf{F}$ can be expressed by

$$
\mathbf{F}=\left[\begin{array}{ccc}
\alpha & 0 & 0 \\
0 & \alpha & \alpha R \tau \lambda \\
0 & 0 & \lambda
\end{array}\right]
$$

Due to the incompressibility assumption, it follows immediately that

$$
J=\operatorname{det}(\mathbf{F})=\alpha^{2} \lambda=1
$$

which implies $\alpha=1 / \sqrt{\lambda}$. Then, the left Cauchy-Green tensor $\mathbf{B}$ reads

$$
\mathbf{B}=\left[\begin{array}{ccc}
\lambda^{-1} & 0 & 0 \\
0 & \lambda^{-1}+\lambda R^{2} \tau^{2} & \sqrt{\lambda^{3}} R \tau \\
0 & \sqrt{\lambda^{3}} R \tau & \lambda^{2}
\end{array}\right] \quad \mathbf{B}^{-1}=\left[\begin{array}{ccc}
\lambda & 0 & 0 \\
0 & \lambda & -\sqrt{\lambda} R \tau \\
0 & -\sqrt{\lambda} R \tau & \lambda^{-2}+R^{2} \tau^{2}
\end{array}\right]
$$

The invariants of the left Cauchy-Green tensor B are according to Eq. (3.6)

$$
\begin{aligned}
& I_{1}=\operatorname{tr}(\mathbf{B})=\lambda^{2}+2 \lambda^{-1}+\lambda R^{2} \tau^{2} \quad I_{3}=\operatorname{det}(\mathbf{B})=1 \\
& I_{2}=\frac{1}{2}\left[(\operatorname{tr}(\mathbf{B}))^{2}-\operatorname{tr}\left(\mathbf{B}^{2}\right)\right]=2 \lambda+\lambda^{-2}+R^{2} \tau^{2}
\end{aligned}
$$


The Cauchy stresses read (Rivlin, 1948; Bahreman and Darijani, 2015)

$$
\begin{aligned}
\sigma_{\theta \theta} & =\int_{R}^{A}-2 \lambda \tau^{2} R \frac{\partial W}{\partial I_{1}} d R+2 \lambda \tau^{2} R^{2} \frac{\partial W}{\partial I_{1}} \\
\sigma_{z z} & =\int_{R}^{A}-2 \lambda \tau^{2} R \frac{\partial W}{\partial I_{1}} d R+2\left(\lambda^{2}-\lambda^{-1}\right) \frac{\partial W}{\partial I_{1}}+2\left(\lambda-\lambda^{-2}-\tau^{2} R^{2}\right) \frac{\partial W}{\partial I_{2}} \\
\sigma_{\theta z} & =2 \sqrt{\lambda^{3}} \tau R \frac{\partial W}{\partial I_{1}}+2 \sqrt{\lambda} \tau R \frac{\partial W}{\partial I_{2}}
\end{aligned}
$$

Therefore, the axial force $N$ required for maintaining this deformation can be calculated as

$$
N=2 \pi \int_{0}^{A} \sigma_{z z} r d r
$$

Substitute the strain energy density functions in Table 2 into Eq. (3.9) by considering Eq. (3.8)

$$
\begin{aligned}
& N_{\text {Neo-Hookean }}=\pi A^{2} \mu\left(\lambda-\lambda^{-2}-\frac{\tau^{2} A^{2}}{4}\right) \\
& N_{\text {Yeoh }}=2 \pi A^{2}\left(\lambda-\lambda^{-2}\right)\left[2 C_{10}+2 C_{20}\left(\lambda^{2}+2 \lambda^{-1}-3\right)+A^{2} C_{20} \tau^{2} \lambda\right. \\
& \left.\quad+3 C_{30}\left(\lambda^{2}+2 \lambda^{-1}-3\right)^{2}+C_{30} A^{4} \lambda^{2} \tau^{4}+3 C_{30} A^{2} \lambda \tau^{2}\left(\lambda^{2}+2 \lambda^{-1}-3\right)\right] \\
& \quad-\pi A^{4} \tau^{2}\left[\frac{1}{2} C_{10}+2 C_{20}\left(\lambda^{2}+2 \lambda^{-1}-3\right)+\frac{2}{3} A^{2} C_{20} \tau^{2} \lambda+\frac{3}{2} C_{30}\left(\lambda^{2}+2 \lambda^{-1}-3\right)^{2}\right. \\
& \left.\quad+2 C_{30} A^{2} \lambda \tau^{2}\left(\lambda^{2}+2 \lambda^{-1}-3\right)+\frac{3}{4} C_{30} A^{4} \lambda^{2} \tau^{4}\right] \\
& N_{\text {Isihara }}=2 \pi A^{2}\left(\lambda-\lambda^{-2}\right)\left[2 C_{10}+2 C_{20}\left(\lambda^{2}+2 \lambda^{-1}-3\right)+A^{2} C_{20} \tau^{2} \lambda+A^{2} \lambda^{-1} C_{01}\right] \\
& \quad-\pi A^{4} \tau^{2}\left[\frac{1}{2} C_{10}+2 C_{20}\left(\lambda^{2}+2 \lambda^{-1}-3\right)+\frac{2}{3} A^{2} C_{20} \tau^{2} \lambda+\lambda^{-1} C_{01}\right]
\end{aligned}
$$

For a pure extension test, $\tau=0$ the axial forces for the hyperelastic models read

$$
\begin{aligned}
& N_{\text {Neo-Hookean }}=\pi\left(\lambda-\lambda^{-2}\right) A^{2} \mu \\
& N_{\text {Yeoh }}=2 \pi\left(\lambda-\lambda^{-2}\right) A^{2}\left[C_{10}+2 C_{20}\left(\lambda^{2}+2 \lambda^{-1}-3\right)+3 C_{30}\left(\lambda^{2}+2 \lambda^{-1}-3\right)^{2}\right] \\
& N_{\text {Isihara }}=2 \pi\left(\lambda-\lambda^{-2}\right) A^{2}\left[C_{10}+2 C_{20}\left(\lambda^{2}+2 \lambda^{-1}-3\right)+\lambda^{-1} C_{01}\right]
\end{aligned}
$$

For a pure torsion test, $\lambda=1$, the axial forces read

$$
\begin{aligned}
& N_{\text {Neo-Hookean }}=-\pi \tau^{2} A^{4} \frac{\mu}{4} \\
& N_{\text {Yeoh }}=-\pi \tau^{2} A^{4}\left(\frac{C_{10}}{2}+\frac{2}{3} C_{20} \tau^{2} A^{2}+\frac{3}{4} C_{30} \tau^{4} A^{4}\right) \\
& N_{\text {Isihara }}=-\pi \tau^{2} A^{4}\left(\frac{C_{10}}{2}+\frac{2}{3} C_{20} \tau^{2} A^{2}+C_{01}\right)
\end{aligned}
$$

Figure 6 shows the solution to Eqs. (3.10)-(3.12) for a cylinder with the initial radius $A=1 \mathrm{~mm}$ and length $L=10 \mathrm{~mm}$. As expected, the different models show different nonlinear responses. In the pure extension case shown in Fig. 6a, the Yeoh and Isihara models, although their mathematical formulations differ, show almost the same behavior. This is because the material parameters of them were determined by the same axial tensile test (Yang et al., 2018). In the 
pure torsion case indicated by Fig. 6d, all models show a negative deformation response, which means the cylinder tends to elongate when twisted. The Neo-Hookean and the Yeoh model show roughly the same responses, while the Isihara model shows much higher reaction forces than the other two models. From the mathematical viewpoint, as shown in Eqs. (3.12), the Neo-Hookean and the Yeoh model contain the first invariant only. The coefficient $C_{01}$ of the second invariant $I_{2}$ in the Isihara model dominates in the torsion case, resulting in large discrepancies. Note that in the torsion case, the twisting deformation is mainly determined by the second invariant. In order

(a)

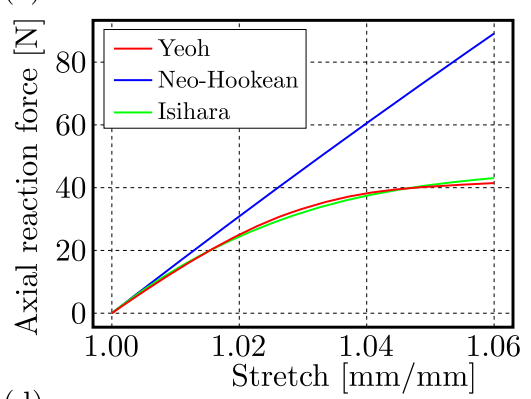

(d)

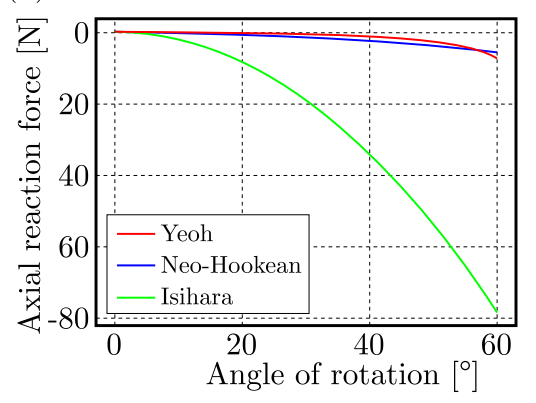

(b)

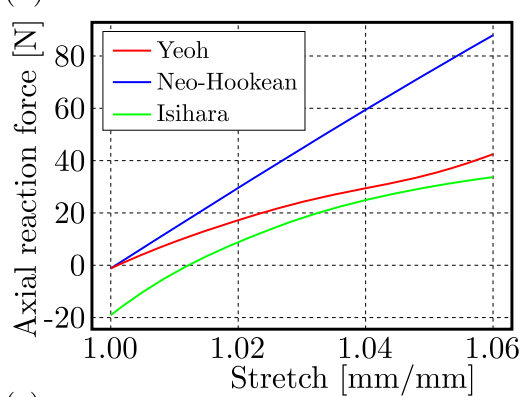

(e)

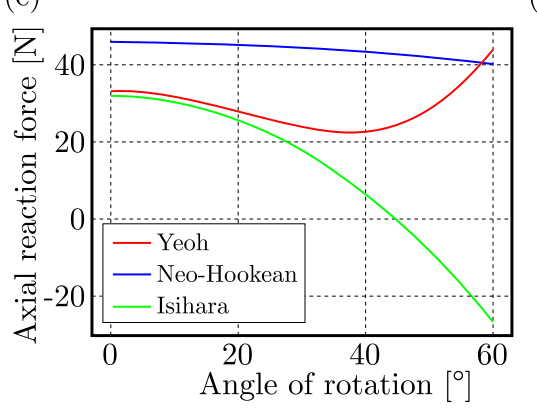

(c)

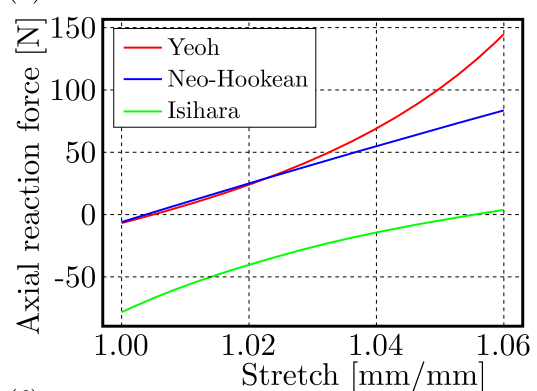

(f)

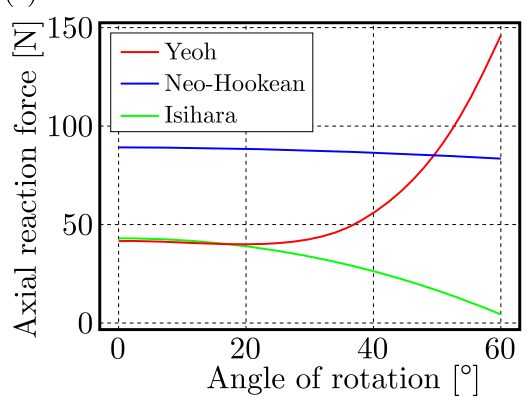

Fig. 6. Axial reaction forces under extension and torsion for a solid cylinder of circular cross-section with the initial radius $A=1 \mathrm{~mm}$ and length $L=10 \mathrm{~mm}$. Axial reaction forces under: (a) pure

extension, (b) extension for the cylinder firstly twisted by $30^{\circ}$, (c) extension for the cylinder firstly twisted by $60^{\circ},(\mathrm{d})$ pure torsion, (e) torsion for the cylinder firstly stretched by 1.03 , (f) torsion for the cylinder firstly stretched by 1.06

to quantify the variances in the reaction force calculations by using further different constitutive laws, the cylinder under both stretch and torsion is considered. Figures $6 \mathrm{~b}$ and $6 \mathrm{c}$ illustrate the axial reaction responses of the cylinder that is first twisted by $30^{\circ}$ and $60^{\circ}$ and afterwards subjected to stretch. All of the hyperelastic models lead to a monotonic increase. Figures 6e and $6 f$ describe the axial reaction responses of the cylinder, first stretched by 1.03 and 1.06 and afterwards subjected to torsion. Despite the fact that the material coefficients were calibrated by the same experimental data, the adopted constitutive laws showed a large discrepancy. We may say that the difference of the responses of these hyperelastic models could be reduced by calibrations using experimental data from a range of experimental tests (uniaxial, biaxial, and planar tension). However, due to the apparent diversity of mathematical forms, it is difficult to eliminate these differences. Because of the great influences of the constitutive laws on the material response, different constitutive laws will be used in the next Section for simulation and assessment of the experiments presented in Section 2. It is expected that some of these hyperelastic models will show a good agreement with the experimental results.

\section{Direct numerical simulation}

For a domain $\Omega \subset \mathbb{R}^{3}$ under Dirichlet boundary conditions, where the displacement is prescribed, or under Neumann boundary conditions, where the traction vector $\widehat{t}_{i}$ is prescribed, we assume 
that the inertial forces as well as the weight are negligible. After a variational formulation, we obtain the weak form (Abali et al., 2017)

$$
\mp=\int_{\Omega} \frac{\partial w}{\partial u_{i, j}} \delta u_{i, j} d V-\int_{\partial \Omega} \widehat{t}_{i} \delta u_{i} d A
$$

The latter integral form vanishes, $\mp=0$, in the case of correct displacements $\mathbf{u}$. However, it is nonlinear in the displacements so that for finding the solution we need to linearize it by using the Newton-Raphson formalism. The solution is found in an incremental manner

$$
\mathbf{u}:=\mathbf{u}+\Delta \mathbf{u}
$$

where the numerical value of $\mathbf{u}$ is overwritten in each increment by $\mathbf{u}+\Delta \mathbf{u}$. By using standard argumentation for linearization, we obtain

$$
\mp+\mathbf{J} \cdot \Delta \mathbf{u}=0
$$

with the Jacobian $\mathbf{J}=\partial \mp / \partial \mathbf{u}$ and the Gateaux derivative

$$
\mathbf{J} \cdot \Delta \mathbf{u}=\lim _{\varepsilon \rightarrow 0} \frac{d}{d \varepsilon} \mp(\mathbf{u}+\varepsilon \Delta \mathbf{u})
$$

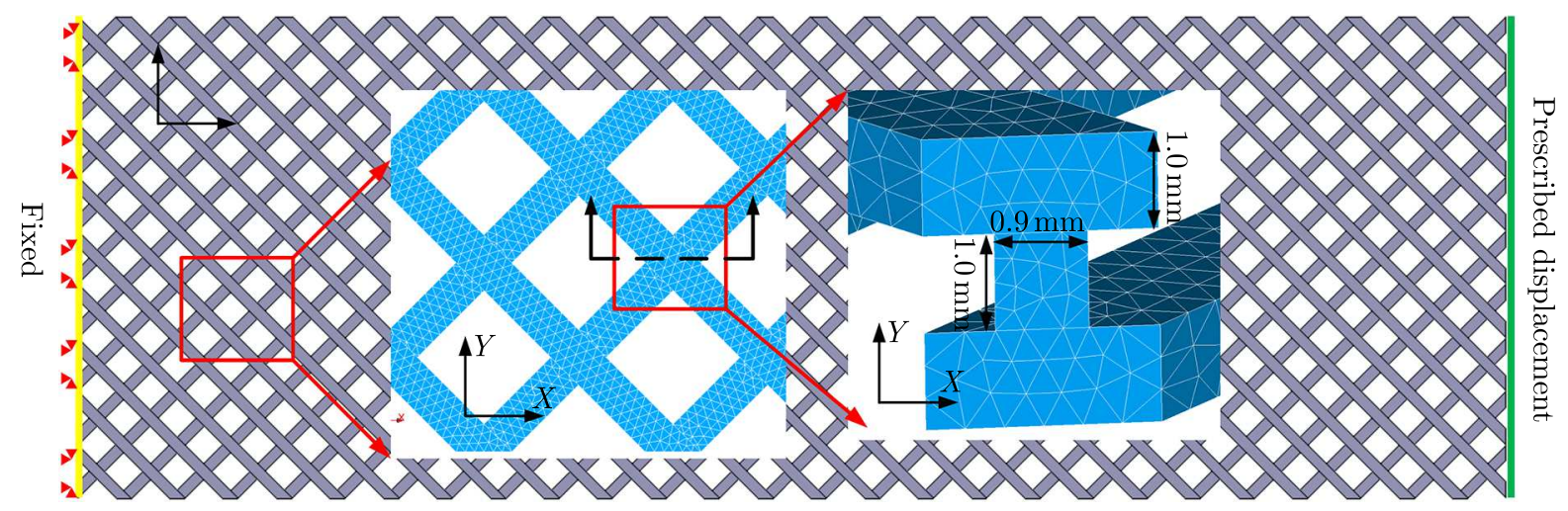

Fig. 7. Boundary conditions and the generated mesh for calculations

The variational equations above are solved using Python programming language and by exploiting FEniCS. The CAD models of the pantographic samples were created on the open source platform SALOME 7.0, and FEM discretizations of the CAD models were realized by the mesh generator NetGen built in SALOME 7.0. FEniCS then assembled the discretized system of FEM equations. As shown in Fig. 7, tetrahedron elements are used to discretize the CAD model, and in order to ensure smoothness and accuracy of the deformation field, Lagrange elements with quadratic basis functions (P2 elements) are used to approximate the solution. One side of the surfaces of the pantographic structure are fixed, namely, three translational degrees of freedom are constrained. The other side surfaces are subjected to prescribed displacements from 0 to $70 \mathrm{~mm}$ with a time step of $1 \mathrm{~mm}$ or a rotation from $0^{\circ}$ to $180^{\circ}$ with a time step $1^{\circ}$. It should be noted that the simulation is quasi-static and the solution from the previous step is reused as the initial value for the next step calculation. The reaction force in the $Y$-direction (shear test) or in the $X$-direction (torsion test) of the side with the applied prescribed displacements or rotations are calculated by summing up the nodal force attached to the surface. Note that a fine mesh is needed for a reasonable approximation of the pantographic structure. After standard h-convergence analysis, which means increasing the degrees of freedom (DOFs) by decreasing the mesh size of each tetrahedron element, 5 million DOFs were used for the simulations discussed in the next Section. 


\section{Comparison of computations with experiments}

Numerical simulations for the experiments undertaken in Section 2 were performed as shown in Fig. 8. It should be noted that FEniCS is able to conduct calculations involving such huge displacements. Therefore, comparison becomes possible by using the measured reaction force as the prescribed displacement is applied. All computational results are summarized in Figs. 9 and 10. Simulations based on the physically linear and geometrically nonlinear St. VenantKirchhoff model were also implemented and used as the reference model to be compared with the experiments and other hyperelastic models.
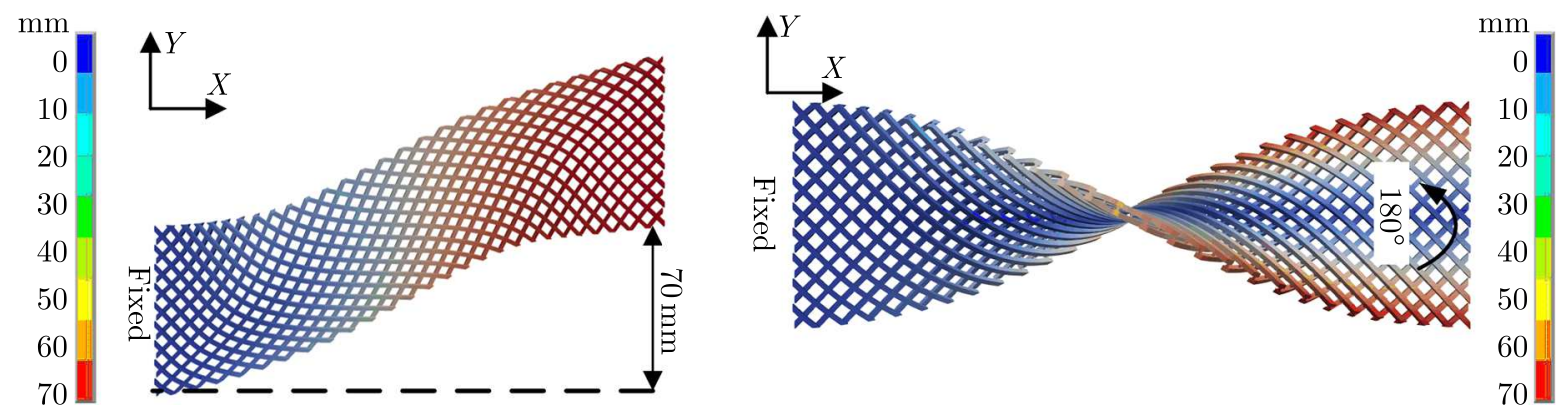

Fig. 8. Simulation results for shear and torsion of the pantographic structure

Sample A

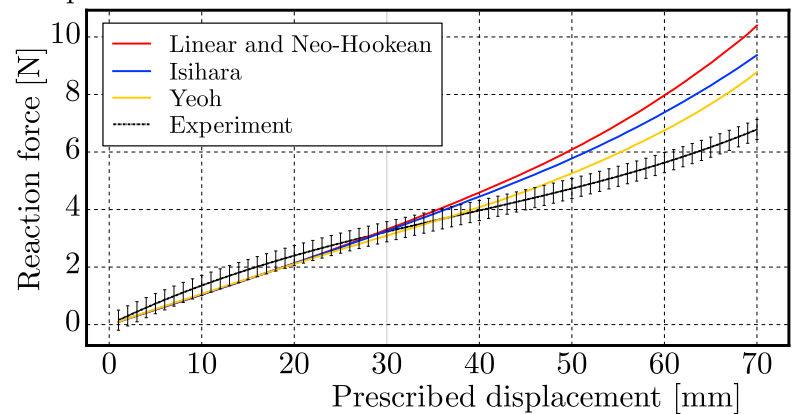

Sample B

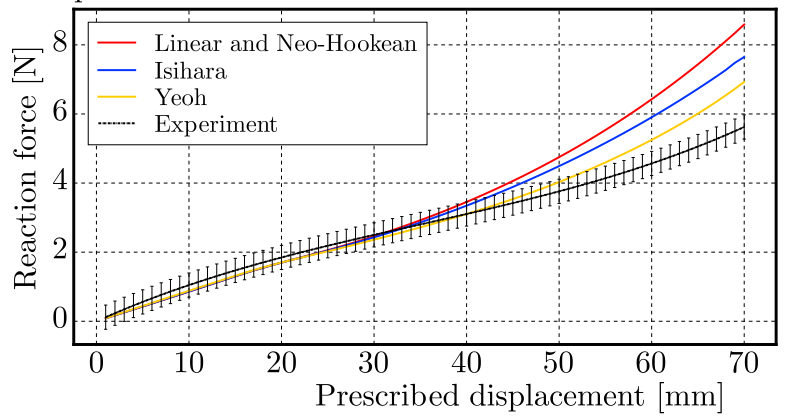

Sample C

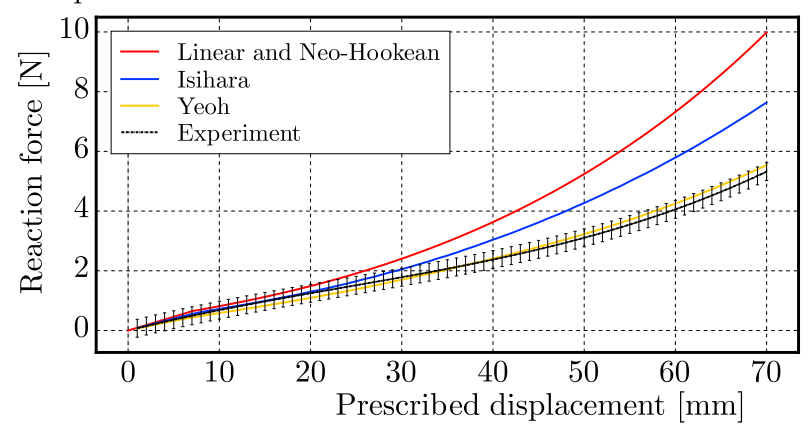

Fig. 9. Reaction force in the displacement controlled shear experiment: experiments versus numerical simulations

Figures 9 and 10 give the results for shear tests and torsion tests, the black dash lines show the experimental reaction forces in the $Y$-direction versus the shearing displacement, and the longitudinal axial reaction forces $(X$-direction) versus the twisting angle, respectively. Obviously, when varying the internal geometric parameters, the samples show quite different nonlinear behavior, and a small variation of the micro-dimensions leads to a remarkable change in the macro-behavior. It is can be seen from Figs. 9 and 10 that, as expected, the linear St. Venant- 

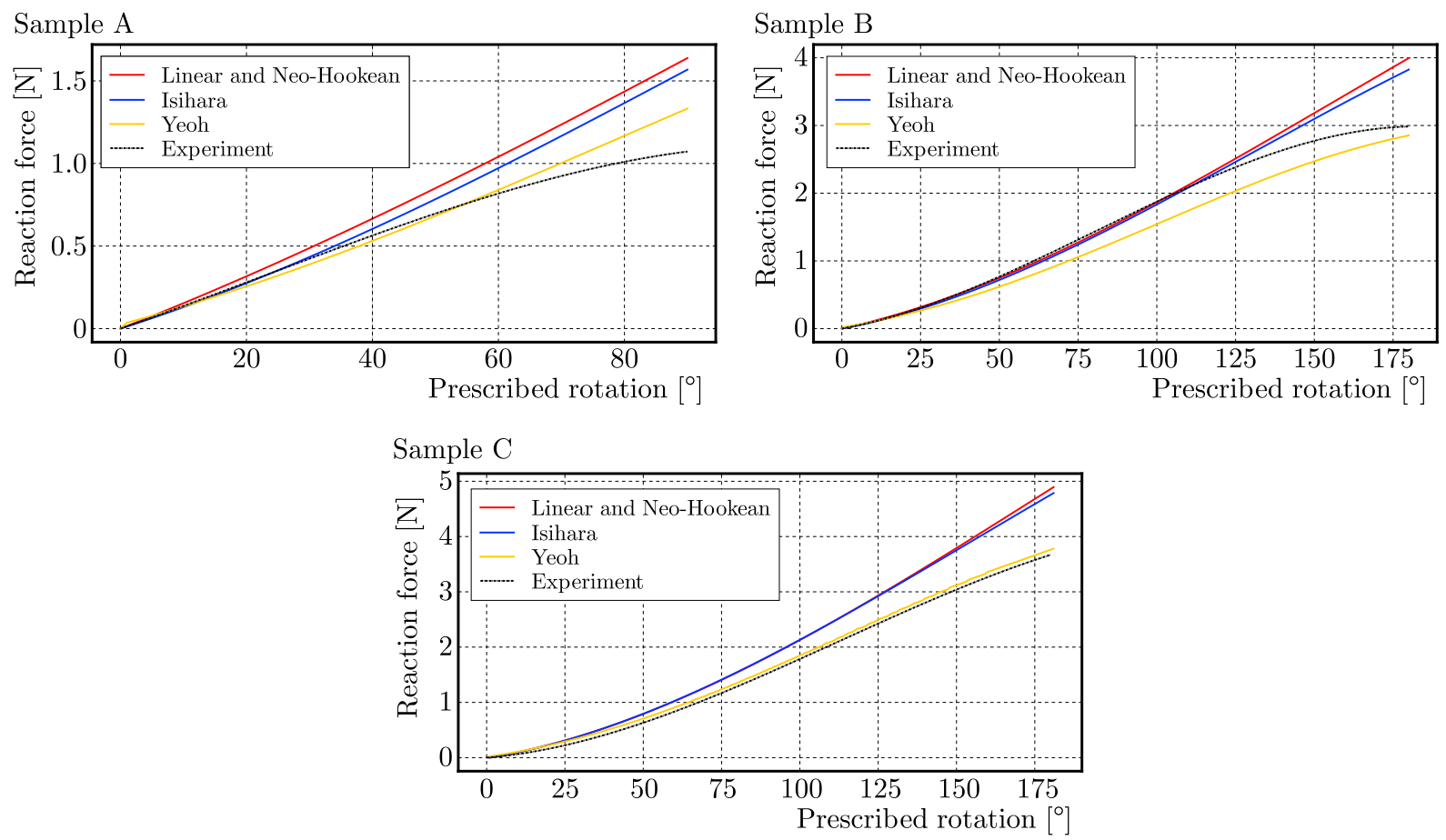

Fig. 10. Reaction force in the rotation controlled torsion experiment: experiments versus numerical simulations

-Kirchhoff model (linear) and other hyperelastic models show good consistency. They all fit the experimental results well in the initial stage. The Neo-Hookean model generates the same result as the linear St. Venant-Kirchhoff model, although their mathematical functions of strain energy differ.

In the shear tests, each sample was tested three times and the results are denoted by the black dash line together with an error bar indicating the error or uncertainty in the measurements. The Isihara model and the Yeoh model show a better match with the experiments than the linear and the Neo-Hookean model. The Yeoh model shows an even better match to the experiments up to $45 \mathrm{~mm}$ (sample A), $50 \mathrm{~mm}$ (sample B), $70 \mathrm{~mm}$ (sample C) of the prescribed displacements. The difference between the Isihara model and Yeoh model results from their different mathematical formulations, as discussed in Section 3. The material parameters were evaluated by a mono-axial tensile test, thus we failed to identify accurately the material coefficients responsible for shearing deformation, mainly given by $I_{2}$. The reason why the Yeoh model fits the experiment better in sample $\mathrm{C}$ than in $\mathrm{A}$ and $\mathrm{B}$ lies in the deformation mechanisms of the pantographic substructure and their underlying energetic contributions. Actually, the deformation energies of pantographic structures mainly consist of three parts (Giorgio et al., 2017), the stretching deformations of the beams, their bending deformations, and the shear distortion related to the variation of the angle between the beams when the pivots are twisted. The responses of the pantographic structures are affected by the relation between the energies associated with the different deformation modes, and these deformation energies depend on the geometrical parameters of the structure shown in Table 1. The three kinds of deformation mechanisms compete during the whole experiments. In the case of sample A and $\mathrm{B}$, the beams mainly resist stretching in the early stages, in other words, the stretching energy of the beams appears to be dominant. With increasing the applied displacements, the beams bend heavily, which also leads to relative rotation of the pivots resulting in the shearing energy. The energies from the shearing of the pivots and the bending of the beams can no loner be ignored. However, the Yeoh model does not incorporate the second invariant, mainly accounting for shearing deformation. Thus the 
Yeoh model fails to reproduce the responses of the overall pantographic structures accurately and quantitatively. As a consequence, a deviation of the Yeoh model and experimental measurements appears. Consider the case of sample $\mathrm{C}$, which is composed of the softest beams when compared to the other samples; because the torsional stiffness of the short pivots is relatively high the rotation between different beams is suppressed and the beams bend easily. However, due to a relatively small length of the beam cross-section sizes, the torsional energy and the bending energy are negligible when compared to the energy coming from the stretching of beams. In such a case, the stretching energy is dominant, and the Yeoh model shows a good match with the measurements.

In the torsion tests, the data were just measured once. Some unavoidable errors could exist due to machine compliance of the mounting device as well as the manufacturing-process of the specimen. Indeed, during the twisting of the specimen, the beams stretched, warped and twisted into helical shape resulting in the shear of the pivots, and the whole structures tended to contract by the external twisting which led to reaction forces in the axial direction $(X$-direction as shown in Fig. 8). In the case of stiffest beams (sample A), since the beams tend to stretch with the twisting of the whole structures in the initial stage, the Yeoh model is able to predict the responses of the pantographic structure up tp 60 degrees. After that bending, twisting energies of the beams and shearing energies of the pivots show up, and this causes deviation of the Yeoh model. In the intermediate case (sample B), the behavior is more complex since the energies from stretching, bending and twisting of the beams as well as the shearing of the pivots coexist during the whole experiment. In the Yeoh model, although showing the same variation tendency, they are smaller than in the experimental curve, especially after rotation of 25 degrees. In the case of the softest beams (sample C), similar to the shear tests, the stretching energies of the beams dominate, thus the Yeoh model shows a good agreement with the experiments. It is should be noted that a combination of different tests would result in the Isihara model parameters matching the experiment better, since the Isihara model contains the second invariant and has the ability to account for the deformation energies coming from stretching, bending, shearing as well as twisting.

\section{Conclusions}

In this paper, the deformation behavior of pantographic structures with different internal geometry parameters were investigated. Shear and torsion experiments for the pantographic structures were outlined and computational modeling was performed by using hyperelastic models under the open-source platform FEniCS. For a better understanding of the deformation responses of the hyperelastic models, analytical solutions for a cylinder under extension and torsion were presented. The comparison between the numerical and the experimental results for pantographic structures were carried by using reaction forces and displacements. The results showed that the linear St. Venant-Kirchhoff model and the Neo-Hookean model provided a good match with the experiments under small deformations for all of the three samples. On the other hand, the Yeoh model, whose coefficients were simply calibrated by a uniaxial test, could predict the response of the pantographic structure quantitatively under the condition that the stretching energy of the beams dominated, resulting from soft beams and stiff pivots. When the bending and shearing deformation energies were not negligible, other models, for example the Isihara model, were required, where more experiments, such as multi-axial tests, pure shear test, etc., were needed to calibrate its coefficients further. We conclude by saying that the study of the responses at microscopic length scale could lead to a possible explanation of an unconventional macroscopic material response. 


\section{References}

1. Abali B.E., Müller W.H., Dell'Isola F., 2017, Theory and computation of higher gradient elasticity theories based on action principles, Archive of Applied Mechanics, 87, 9, 1495-1510

2. Altenbach H., Eremeyev V.A., 2012, Generalized Continua - from the Theory to Engineering Applications, 541, Springer

3. Bahreman M., Darijani H., 2015, New polynomial strain energy function; application to rubbery circular cylinders under finite extension and torsion, Journal of Applied Polymer Science, 132, 13, 41718

4. Barchiesi E., Ganzosch G., Liebold C., Placidi L., Grygoruk R., Müller W.H., 2018a, Out-of-plane buckling of pantographic fabrics in displacement-controlled shear tests: experimental results and model validation, Continuum Mechanics and Thermodynamics, 1-13, DOI: $10.1007 / \mathrm{s} 00161-018-0626-\mathrm{x}$

5. Barchiesi E., Spagnuolo M., Placidi L., 2018b, Mechanical metamaterials: a state of the art, Mathematics and Mechanics of Solids, 1-23, DOI: 10.1177/1081286517735695

6. Brodecki A., Szymczak T., Kowalewski Z., 2018, Digital image corellation technique as a tool for kinematics assessments of structural components, Acta Mechanica et Automatica, 12, 2, 101-104

7. Chen J.-S., Wu C.-T., 1997, On computational issues in large deformation analysis of rubber bushings, Journal of Structural Mechanics, 25, 3, 287-309

8. Del Vescovo D., Giorgio I., 2014, Dynamic problems for metamaterials: review of existing models and ideas for further research, International Journal of Engineering Science, 80, 153-172

9. Dell'Isola F., Andreaus U., Placidi L., 2014, At the origins and in the vanguard of peridynamics, non-local and higher-gradient continuum mechanics: An underestimated and still topical contribution of Gabrio Piola, Mathematics and Mechanics of Solids, 20, 887-928

10. Dell'Isola F., Giorgio I., Pawlikowski M., Rizzi N.L., 2016, Large deformations of planar extensible beams and pantographic lattices: heuristic homogenization, experimental and numerical examples of equilibrium, Proceedings of the Royal Society A, 472, 2185, 1-23

11. Dell'Isola F., Lekszycki T., Pawlikowski M., Grygoruk R., Greco L., 2015, Designing a light fabric metamaterial being highly macroscopically tough under directional extension: first experimental evidence, Zeitschrift für angewandte Mathematik und Physik, 66, 6, 3473-3498

12. Ganzosch G., Dell'Isola F., Turco E., Lekszycki T., Müller W.H., 2016, Shearing tests applied to pantographic structures, Acta Polytechnica CTU Proceedings, 7, 1-6

13. Ganzosch G., Hoschke K., Lekszycki T., Giorgio I., Turco E., Müller W.H., 2018, 3D-measurements of 3D-deformations of pantographic structures, Technische Mechanik, 38, 3, 233-245

14. Holzapfel G.A., 2000, Nonlinear Solid Mechanics: a Continuum Approach for Engineering Science, Wiley, Chichester

15. Kowalewski Z.L., Szymczak T., Maciejewski J., 2014, Material effects during monotonic-cyclic loading, International Journal of Solids and Structures, 51, 3-4, 740-753

16. Lam D., Yang F., Chong A., Wang J., Tong P., 2003, Experiments and theory in strain gradient elasticity, Journal of the Mechanics and Physics of Solids, 51, 8, 1477-1508

17. Lawlor M.G., O’Donnell M.R., O'Connell B.M., Walsh M.T., 2011, Experimental determination of circumferential properties of fresh carotid artery plaques, Journal of Biomechanics, 44, 9, 1709-1715

18. Logg A., Mardal K.A., Wells G.N., 2011, Automated solution of differential equations by the finite element method, the FEniCS book, Lecture Notes in Computational Science and Engineering, 84, Springer 
19. Mindlin R.D., Eshel N.N., 1968, On first strain-gradient theories in linear elasticity, International Journal of Solids and Structures, 4, 1, 109-124

20. Misra A., Lekszycki T., Giorgio I., Ganzosch G., Müller W.H., dell'Isola F., 2018, Pantographic metamaterials show atypical Poynting effect reversal, Mechanics Research Communications, 89, 6-10

21. RIVLIn R.S., 1948, Large elastic deformations of isotropic materials. III. Some simple problems in cyclindrical polar co-ordinates, Philosophical Transactions of the Royal Society A, 240, 823, 509-525

22. Thai H.-T., Vo T.P., Nguyen T.-K., Kim S.-E., 2017, A review of continuum mechanics models for size-dependent analysis of beams and plates, Composite Structures, 177, 196219

23. Toupin R.A, 1964, Theories of elasticity with couple-stress, Archive for Rational Mechanics and Analysis, 17, 2, 85112

24. Yang H., Ganzosch G., Giorgio I., Abali B.E., 2018, Material characterization and computations of a polymeric metamaterial with a pantographic substructure, Zeitschrift für angewandte Mathematik und Physik, 69, 4, 105

Manuscript received September 16, 2018; accepted for print January 10, 2019 